\title{
In memoriam Giorgio Bronz (1947-2018)
}

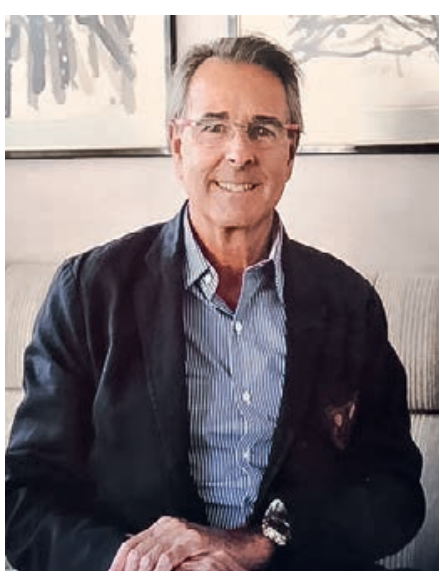

Giorgio Bronz

Lo scorso 29 luglio si è spento inaspettatamente il Dr. Giorgio Bronz, primo presidente e socio fondatore della Società Ticinese di Chirurgia Plastica, Ricostruttiva ed Estetica.

Il Dr. Bronz, specialista in chirurgia plastica, ricostruttiva ed estetica ed in chirurgia generale, dal 1984 operava in Ticino nella sua clinica di Cassarate, nelle cliniche Santa Chiara, Sant'Anna e Ars Medica, di cui è stato promotore.

Era stato consulente all'EOC e al Centro Cantonale di Senologia, di cui era stato socio fondatore.

Corrispondenza: Dr. med. Francesca Casellini Ghiringhelli

Specialista CH/FMH in chirurgia plastica ricostruttiva ed estetica

Via Nassa 7, 6900 Lugano Tel. +41919212784 studio.dr.fcg[at]ticino.com
L'ISAPS (Società Internazionale di Chirurgia Plastica Estetica) gli aveva conferito il titolo di professore per l'insegnamento postuniversitario di chirurgia plastica ed estetica.

Il Dr. Bronz si era adoperato per fondare la nostra società, la STCPRE, con lo scopo di difendere gli interessi professionali, curare la collegialità negli anni in cui si sono intensificati i diritti di pratica in Svizzera, con attenzione alla formazione, all'etica, al codice deontologico della FMH e della Società Svizzera di Chirurgia Plastica, Ricostruttiva ed Estetica.

Il Dr. Bronz amava la sua professione, la bellezza, l'arte della chirurgia estetica, l'armonia dei tratti, degli elementi.

Lo ricordiamo, sempre impeccabile, nel suo studio progettato dall'architetto Botta, fra le sue opere d'arte, i suoi quadri, le sue sculture, la sua musica.

Amava la natura, le montagne; era un provetto sciatore ed appassionato hockeista.

Rammentava le sue origini, la sua infanzia ad Airolo ed i momenti trascorsi nello studio medico del padre, dove fin da bambino aveva amato la professione.

Ricercava la bellezza in tavola, in cucina, nell'arte, nella natura, negli abiti, nella chirurgia; sensibilità acquisite dalla mamma.

Un uomo appassionato ed instancabile lavoratore. Sottolineava che il suo lusso più grande era la sua famiglia, il suo lavoro, la sua casa.

S'illuminava quando parlava di sua moglie Isabella e dei suoi figli Gregory, Géraldine e Jennifer.

A loro, alla mamma, al fratello e collega Lucio, ai famigliari, va il nostro pensiero.

per la STCPRE: Francesca Casellini Ghiringhelli con Sandra Baldi Balmelli, Damir De Monaco, Paolo Erba, Philipp Fallscheer, Yves Harder, Giacomo Martinoni, Martino Meoli 